ARTIGO ORIGINAL

\title{
Efeito de rotações sucessivas de eucalipto na mineralização de nitrogênio e carbono do solo e suprimento de nitrogênio, no Sudoeste da Bahia, Brasili
}

\author{
Effect of successive rotations of eucalyptus on nitrogen and carbon \\ mineralization of the soil and nitrogen supply in the Southwest of Bahia, Brazil
}

Ariane Miranda de Oliveira' (D), Patrícia Anjos Bittencourt Barreto-Garcia' (i), Bruno José Rodrigues Alves² (D), Valdemiro Conceição Júnior ${ }^{1}$ (D), Emanuela Forestieri Gama-Rodrigues ${ }^{3}$ (i)

'Universidade Estadual do Sudoeste da Bahia - UESB, Vitória da Conquista, BA, Brasil

${ }^{2}$ Empresa Brasileira de Pesquisa Agropecuária - Embrapa Agrobiologia, Seropédica, RJ, Brasil

${ }^{3}$ Universidade Estadual do Norte Fluminense Darcy Ribeiro - UENF, Campos dos Goytacazes, RJ, Brasil

Como citar: Oliveira, A. M., Barreto-Garcia, P. A. B., Alves, B. J. R., Conceição Júnior, V., \& Gama-Rodrigues, E. F. (2020). Efeito de rotações sucessivas de eucalipto na mineralização de nitrogênio e carbono do solo e suprimento de nitrogênio, no Sudoeste da Bahia, Brasil. Scientia Forestalis, 48(126), e3346.

https://doi.org/10.18671/scifor.v48n126.15

\begin{abstract}
Resumo
O objetivo deste estudo foi avaliar o efeito de rotações sucessivas de cultivo de eucalipto sobre: (a) as reservas orgânicas de nitrogênio e carbono potencialmente mineralizáveis do solo e (b) o suprimento de nitrogênio por estas reservas em plantações de Eucalyptus urophylla que não receberam adubação nitrogenada. O estudo foi conduzido em plantações comerciais de Eucalyptus urophylla em primeira, segunda e terceira rotação, tendo como referência uma área de Floresta Estacional Decidual Submontana. Foram alocadas quatro unidades experimentais de $400 \mathrm{~m}^{2} \mathrm{em}$ cada área, onde realizaram-se coletas de amostras de solo deformadas e indeformadas, de serapilheira acumulada e de biomassa arbórea. A partir das quantidades de nitrogênio e carbono mineralizados durante 24 semanas de incubação, foram estimados o potencial de mineralização de $\mathrm{N}$ e $\mathrm{C}$ dos solos. Além disso, foram determinados os teores de $\mathrm{N}$ total do solo, da serapilheira e da biomassa arbórea. Para estimar a participação das reservas orgânicas no suprimento de $\mathrm{N}$ para as plantações de eucalipto foram adotados dois modelos de predição. Os solos sob eucalipto em diferentes rotações e floresta nativa possuem capacidade similar de mineralizar N e C, com potenciais de mineralização médios de 47.6 e 597.2 mg kg-1 de solo, respectivamente. O potencial de mineralização de nitrogênio e de carbono do solo não é alterado pelo cultivo de eucalipto em até três rotações consecutivas. As reservas orgânicas de nitrogênio do solo, de acordo com os modelos de predição, são suficientes para atender a demanda de $\mathrm{N}$ pelo eucalipto por um período de três a oito rotações.
\end{abstract}

Palavras-chave: Eucalyptus urophylla; N mineralizado; Matéria orgânica; Ciclagem de nutrientes.

\begin{abstract}
The aim of this study was to evaluate the effect of successive rotations of eucalyptus cultivation on: (a) organic reserves of nitrogen and carbon potentially mineralizable of the soil and (b) the nitrogen supply from these reserves in Eucalyptus urophylla plantations without nitrogen fertilization. The study was conducted in commercial plantations of Eucalyptus urophylla on the first, second and third rotation,
\end{abstract}

'Parte da Dissertação de Mestrado da primeira autora, apresentada à Universidade Estadual do Sudoeste da Bahia UESB, pelo Programa de Pós-Graduação em Ciências Florestais.

Fonte de financiamento: Fundação de Amparo à Pesquisa do Estado da Bahia (FAPESB) por meio da concessão de bolsa de Mestrado (termo $\mathrm{n}^{\circ}$

0187/2017). Conflito de interesse: Nada a declarar.

Autor correspondente: patriciabarreto@uesb.edu.br

Recebido: 23 abril 2019.

Aceito: 19 julho 2019

Editor: Paulo Henrique Müller Silva.

(c) Este é um artigo publicado em acesso aberto (Open Access) sob a licença Creative Commons Attribution, que permite uso, distribuição e reprodução em qualquer meio, sem restrições desde que o trabalho original seja corretamente citado. 
with reference to Submontane Deciduous Seasonal Forest area. Four experimental units of $400 \mathrm{~m}^{2}$ were allocated in each area, where samples of deformed and undisturbed soil samples were collected, accumulated litter and tree biomass. From the nitrogen and carbon mineralized amounts during 24 weeks of incubation, the $\mathrm{N}$ and $\mathrm{C}$ mineralization potential of the soils was estimated. In addition, total soil $\mathrm{N}$, litter and tree biomass levels were determined. To estimate the share of organic reserves in the $\mathrm{N}$ supply for eucalyptus plantations, two prediction models were adopted. Soils under eucalyptus by different rotations and native forest have been similar mineralization capacity of $\mathrm{N}$ and $\mathrm{C}$, with average mineralization potentials of 47.6 and $597.2 \mathrm{mg} \mathrm{kg}^{-1}$ of soil, respectively. The nitrogen and carbon mineralization potential of the soil were not altered by eucalyptus cultivation in up to three consecutive rotations. The nitrogen organics reserves of the soil, according to the prediction models, are sufficient to provide the demand of $\mathrm{N}$ by eucalyptus between three to eight rotations.

Keywords: Eucalyptus urophylla; N mineralized; Organic matter; Nutrient cycling.

\section{INTRODUÇÃO}

No Brasil, as plantações com o gênero Eucalyptus spp. ocupam uma área correspondente a cerca de 5.7 milhões de hectares e se encontram distribuídas nas diferentes regiões do país (Indústria Brasileira de Árvores, 2017). No estado da Bahia, as áreas plantadas com o gênero correspondem, atualmente, a 612 mil hectares (Indústria Brasileira de Árvores, 2017) e estão em progressiva expansão, inclusive para regiões com solos de baixa fertilidade e restrições na quantidade e distribuição de chuvas (Fernandes et al., 2018).

Na região Sudoeste do estado da Bahia, a maior parte das plantações de eucalipto são voltadas para a produção de biomassa energética, adotando-se rotações curtas (entre 4 e 6 anos) e suplementação nutricional insuficiente, já que normalmente são empregadas apenas adubações fosfatadas. Tal condição pode comprometer a produtividade futura do sítio florestal.

Dentre os elementos essenciais, o nitrogênio $(\mathrm{N})$ é um dos mais requeridos pelo eucalipto e, por ser exportado em grandes quantidades, costuma demandar suplementação de $N$ por meio de adubos nitrogenados. No entanto, observa-se que o eucalipto apresenta baixa resposta à fertilização nitrogenada (Barros et al., 1990) ou ainda bom desenvolvimento na ausência deste tipo de adubação (Azevedo et al., 2011), o que indica que a mineralização das reservas orgânicas de nitrogênio do solo estariam suprindo a demanda das plantas ao longo do ciclo da cultura (Barreto et al., 2012; Gama-Rodrigues et al., 2005).

Tais indícios também levam à suposição de que, após vários ciclos de cultivo, ocorrerá redução das reservas de $\mathrm{N}$ orgânico potencialmente mineralizável do solo. Como a diminuição do $\mathrm{N}$ do solo está estreitamente relacionada à redução das fontes de $\mathrm{C}$ facilmente mineralizáveis (Gonçalves et al., 2001), pesquisas que associam o estudo da mineralização de nitrogênio com a mineralização de carbono podem constituir um potencial indicador da disponibilidade de $\mathrm{N}$ às plantas (Barreto et al., 2010).

Dentre os principais métodos utilizados para avaliar a mineralização de $\mathrm{N}$ estão as incubações aeróbias em laboratório, que fornecem estimativas satisfatórias da fração potencialmente mineralizável do $\mathrm{N} \mathrm{e}$, ou do $\mathrm{C}$ orgânico do solo. Para determinar esta fração e descrever o padrão de mineralização ao longo do tempo, são empregados diversos modelos matemáticos, estando entre os mais utilizados os do tipo exponencial (Molina et al., 1980; Stanford \& Smith, 1972), linear (Gonçalves et al., 2001) e sigmoidal (Nunes et al., 2016).

Apesar das evidências de que a mineralização de nitrogênio exerce importante papel no suprimento deste elemento em plantações de eucalipto, os estudos realizados até então (Barreto et al., 2010, 2012; Dijkstra et al., 2017; Gonçalves et al., 2001; Pulito et al., 2015; Tchichelle et al., 2017) não permitem maiores considerações sobre a dinâmica de $\mathrm{N}$ no solo após períodos sucessivos de cultivo com o gênero. Dessa forma, objetivou-se com o presente estudo avaliar o efeito de rotações sucessivas de cultivo de eucalipto sobre (a) as reservas orgânicas de nitrogênio e carbono potencialmente mineralizáveis do solo e (b) o suprimento de nitrogênio por essas reservas em plantações de Eucalyptus urophylla que não receberam adubação nitrogenada. 


\section{MATERIAL E MÉTODOS}

\section{Descrição das áreas estudadas}

O estudo foi conduzido em áreas de plantações comerciais de Eucalyptus urophylla S. T. Blake e de floresta nativa estabelecidas sobre Latossolo Amarelo distrófico, de textura argilo-arenosa e baixa fertilidade natural (Tabela 1), localizadas no município de Vitória da Conquista, região Sudoeste do estado da Bahia. Tais plantações são destinadas à produção de madeira para fins energéticos, apresentando ciclos de corte de cinco anos. A região apresenta relevo plano a levemente ondulado e clima classificado como Cwb (tropical de altitude), segundo a classificação de Köppen, com média de temperatura e precipitação anual de $21{ }^{\circ} \mathrm{C}$ e $700 \mathrm{~mm}$, respectivamente.

Tabela 1. Atributos químicos e físicos de solos sob eucalipto em diferentes rotações e floresta nativa na camada $0-10 \mathrm{~cm}$ de profundidade

\begin{tabular}{|c|c|c|c|c|c|c|c|c|c|c|c|c|c|}
\hline \multirow{2}{*}{ Área } & \multirow{2}{*}{ pH } & $\cos$ & $\mathbf{N}_{\mathrm{t}}$ & \multirow{2}{*}{$\frac{P}{m g d m^{-3}}$} & Al & $\mathrm{H}+\mathrm{Al}$ & $\mathbf{K}$ & $\mathrm{Ca}$ & Mg & ARE & SIL & ARG & \multirow{2}{*}{$\frac{\text { Ds }}{\mathrm{g} \mathrm{cm}^{-3}}$} \\
\hline & & \multicolumn{2}{|c|}{$-\mathrm{g} \mathrm{kg}^{-1}-$} & & \multicolumn{5}{|c|}{$---\mathrm{cmol}_{\mathrm{c}} \mathrm{dm}^{-3}-\ldots$} & \multicolumn{3}{|c|}{$-\mathrm{g} \mathrm{kg}^{-1}-$ - } & \\
\hline $\mathbf{R} 1$ & 4,5 & 7,1 & 0,70 & 1,0 & 1,2 & 5,6 & 0,15 & 0,5 & 0,5 & 550 & 80 & 370 & 1,17 \\
\hline $\mathbf{R} 2$ & 4,6 & 7,4 & 0,90 & 1,0 & 0,9 & 6,4 & 0,12 & 0,7 & 0,7 & 470 & 70 & 460 & 1,11 \\
\hline R3 & 4,4 & 8,3 & 0,80 & 2,3 & 1,3 & 6,9 & 0,13 & 0,4 & 0,8 & 455 & 75 & 470 & 1,30 \\
\hline FN & 4,7 & 12,9 & 1,30 & 1,5 & 0,8 & 6,0 & 0,18 & 0,8 & 0,8 & 580 & 50 & 370 & 1,18 \\
\hline
\end{tabular}

R1: Eucalipto em primeira rotação de cultivo; R2: Eucalipto em segunda rotação, totalizando 9 anos de cultivo; R3: Eucalipto em terceira rotação, totalizando 14 anos de cultivo; FN: floresta nativa; COS: C orgânico do solo; $\mathrm{N}$ t: $\mathrm{N}$ total; ARE: areia; SIL: silte; ARG: argila; Ds: densidade do solo. Análises realizadas de acordo com a Empresa Brasileira de Pesquisa Agropecuária (2017): pH (água); P e K extraíveis por Mehlich-1; Ca, Mg e Al trocáveis por KCl $1 \mathrm{~mol} \mathrm{~L}^{-1}$. granulometria pelo método da pipeta e densidade pelo método do anel volumétrico. Foram utilizadas quatro amostras compostas por 20 amostras simples, de cada área de estudo.

As áreas de estudo foram selecionadas de acordo com o tempo a que foram submetidas ao cultivo de eucalipto, de modo a obter uma sequência progressiva de tempo. Assim, foram definidos quatro tratamentos: R1 - eucalipto em primeira rotação de cultivo, área cultivada a quatro anos, estabelecida em regime de alto fuste e espaçamento $3 \mathrm{~m} \times 4 \mathrm{~m}$; R2 - eucalipto em segunda rotação, área cultivada a nove anos, conduzida em regime de talhadia (com a manutenção de dois ou três brotos) e espaçamento de $3 \mathrm{~m} \times 3 \mathrm{~m}$; R3 - eucalipto em terceira rotação de cultivo, área cultivada a catorze anos, também conduzida em regime de talhadia e espaçamento de $3 \mathrm{~m} \times 3 \mathrm{~m}$; e FN - área de referência, localizada nas proximidades das áreas cultivadas com eucalipto, constituída de floresta nativa em estágio médio de regeneração, segundo critérios estabelecidos pela Resolução CONAMA nº. 1, de 31 de Janeiro de 1994 (Brasil, 1994), já que a área não sofre intervenção a mais de 20 anos. A localização das áreas estudadas está apresentada na Figura 1. 


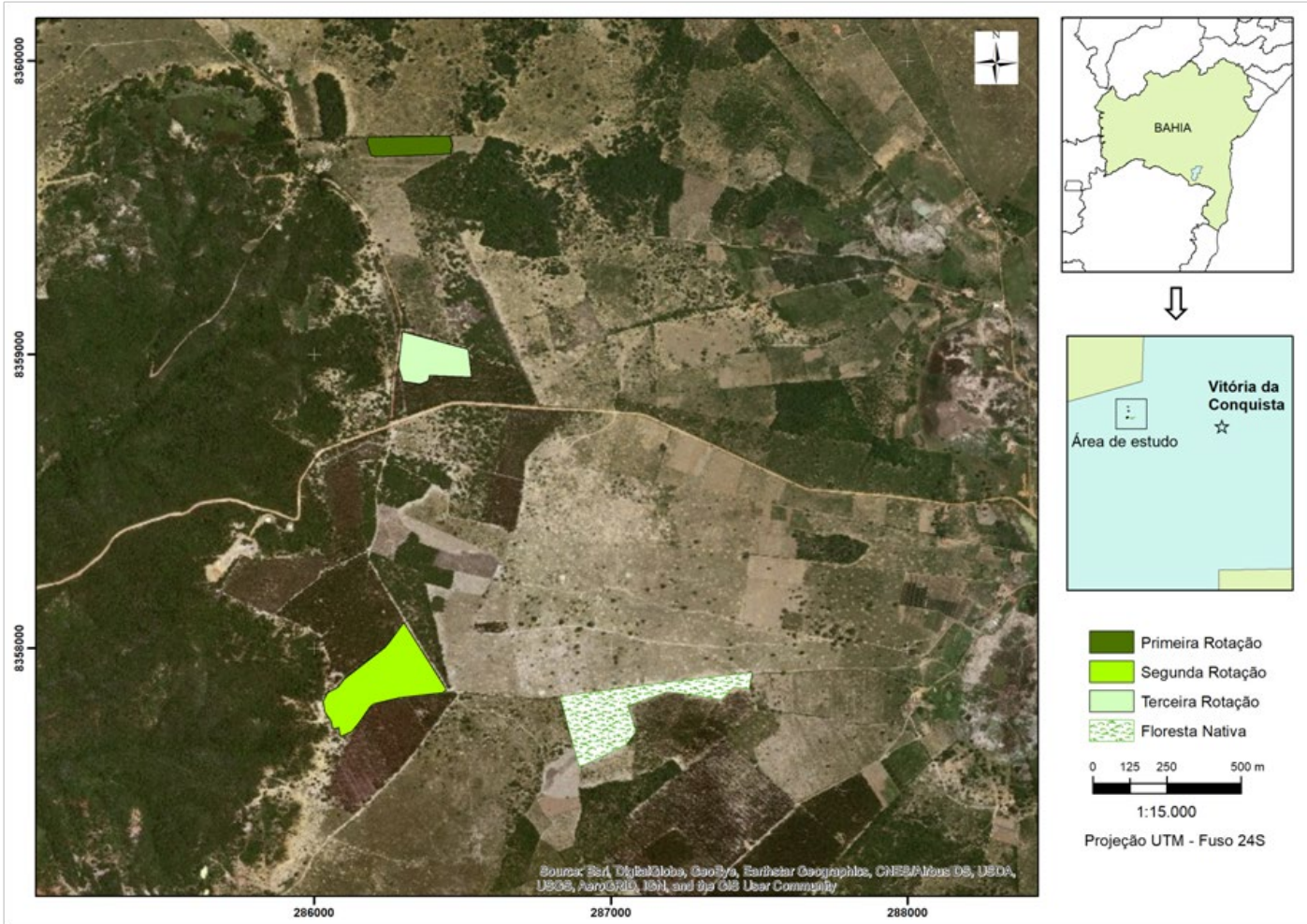

Figura 1. Localização da área e subáreas estudadas no município de Vitória da Conquista, Bahia, Brasil.

O fragmento de floresta nativa possui área total de cerca de 20 hectares e localiza-se em uma zona de transição entre Caatinga e Mata Atlântica. A vegetação é classificada como Floresta Estacional Decidual Submontana. Apresenta adaptações para aridez, com folhagem esclerófila de pequeno tamanho, gemas protegidas por pêlos e acúleos. Trata-se de uma floresta com estrato arbóreo baixo (árvores com altura máxima de $10 \mathrm{~m}$ ) e predominância de espécies da família Fabaceae, sobretudo do gênero Parapiptadenia spp. (Instituto Brasileiro de Geografia e Estatística, 2012).

Os plantios foram realizados a partir de mudas seminais, com subsolagem nas linhas de plantio (50 cm de profundidade) e abertura de covas. As áreas não receberam adubação nitrogenada, apenas adição de superfosfato simples na etapa de plantio (300 g de superfosfato simples por cova), sendo essa uma prática recorrente de pequenos e médios produtores em plantios de eucalipto na região Sudoeste da Bahia. Até os 12 meses após o plantio foi adotado controle de mato-competição, por meio de capinas nas linhas e entrelinhas. Antes de serem cultivadas com eucalipto, as áreas eram ocupadas por pastagens degradadas.

\section{Amostragem do solo, serapilheira e biomassa arbórea}

Em cada área foram estabelecidas quatro parcelas com dimensões de $20 \mathrm{~m} \times 20 \mathrm{~m}$. As amostragens foram realizadas por parcela. No caso do solo, a coleta foi realizada no mês de outubro de 2017, em três camadas: 0-10, 10-20 e 20-40 cm. Em cada uma destas profundidades foram retiradas 20 amostras simples, que foram reunidas formando uma composta, e amostras indeformadas com uso de anéis volumétricos para se estimar a densidade do solo.

Para a coleta de serapilheira acumulada, em cada plantação de eucalipto, foram selecionadas quatro árvores-amostra, representativas do diâmetro à altura do peito (DAP) médio de cada parcela experimental $(\mathrm{R} 1=15.8 ; 11.1 ; 12.0$ e $12.0 \mathrm{~cm}$. $\mathrm{R} 2=9.6 ; 10.1 ; 10.0$ e $10.4 \mathrm{~cm}$. $\mathrm{R} 3=12.5 ; 14.3 ; 10.8$ e $11.9 \mathrm{~cm}$ ). A amostragem foi realizada em dezembro de 2017, a partir da delimitação de uma área de $2 \mathrm{~m} \times 2 \mathrm{~m}$ no entorno do tronco de cada árvore-amostra. O material coletado foi seco $\left(a 5^{\circ} \mathrm{C}\right.$ em estufa de circulação forçada de ar), pesado, triturado em moinho (tipo Wiley) e tamisado em peneira (malha 20 mesh). Os resultados de massa seca (g) obtidos foram convertidos para $\mathrm{Mg} \mathrm{ha}^{-1}$. 
A quantificação da biomassa arbórea foi realizada nas mesmas árvores-amostra selecionadas para a coleta de serapilheira, também em dezembro de 2017, adotando-se o método destrutivo com procedimento de simples separação (Sanquetta et al., 2004). Cada árvore-amostra foi fracionada nos compartimentos folhas, galhos e fuste com casca, que foram pesados em campo com uso de balança mecânica (capacidade de $150 \mathrm{~kg}$ e precisão de $50 \mathrm{~g}$ ). Depois da pesagem, retiraram-se amostras de cada componente, que foram secas a $65^{\circ} \mathrm{C}$ e moídas. A biomassa seca por árvore (kg) foi estimada por meio da multiplicação da massa verde pelo fator de correção de umidade (razão entre a massa seca da amostra e a massa úmida da amostra).

\section{Nitrogênio total e carbono orgânico}

Foram realizadas análises químicas para determinação dos teores de carbono orgânico do solo (COS) (camada 0-10 cm) e dos teores de nitrogênio total $\left(\mathrm{N}_{\text {total }}\right)$ das amostras de serapilheira, biomassa arbórea e solo (camadas 0-10, 10-20 e 20-40 cm). A escolha da profundidade 0-10 $\mathrm{cm}$ para a avaliação do COS foi baseada no fato desta comportar maiores conteúdos de matéria orgânica e maior atividade dos microrganismos do solo. Por sua vez, as análises de $\mathrm{N}$ total foram expandidas até a profundidade de $40 \mathrm{~cm}$ para que os resultados pudessem ser considerados nos modelos de predição do suprimento de $\mathrm{N}$, conforme descrito em tópico a seguir, já que esta camada constitui uma reserva significativa de nitrogênio orgânico potencialmente mineralizável a ser disponibilizado para as árvores. Para as análises de $\mathrm{COS}$ foi adotado o método da oxidação com dicromato de potássio $\left(\mathrm{K}_{2} \mathrm{Cr}_{2} \mathrm{O}_{7}\right) 0.4 \mathrm{~mol} \mathrm{~L}^{-1}$ (Defelipo \& Ribeiro, 1981), e para as de $\mathrm{N}_{\text {total }}$ o método Kjeldahl (Bataglia et al., 1983).

\section{Mineralização de N e C}

A mineralização de nitrogênio $(\mathrm{N})$ e de carbono (C) do solo foi avaliada na profundidade 0-10 cm, em condições aeróbias de laboratório, conforme proposto por Stanford \& Smith (1972) com adaptações de Alves (1989). Foram utilizados $50 \mathrm{~g}$ de solo juntamente com $50 \mathrm{~g}$ de areia lavada e peneirada, sendo esta combinação acondicionada em colunas de percolação (em duplicata), confeccionadas com tubos de PVC com $4 \mathrm{~cm}$ de diâmetro e $30 \mathrm{~cm}$ de altura, que receberam na porção inferior buchas de lã de vidro para reter o solo. Para todo o experimento, seis colunas contendo $100 \mathrm{~g}$ de areia lavada foram utilizadas como controle.

A extração do $\mathrm{N}$ mineralizado ocorreu através da percolação de $100 \mathrm{~mL}$ da solução extratora $\left(\mathrm{CaCl}_{2} 0.01 \mathrm{~mol} \mathrm{~L}^{-1}\right)$, que foi fracionada em cinco apliçções de $20 \mathrm{~mL}$. Após cada extração foi adicionado $50 \mathrm{~mL}$ de solução nutritiva desprovida de $\mathrm{N}$ (duas aplicações de $25 \mathrm{~mL}$ ), composta por $\mathrm{K}_{2} \mathrm{SO}_{4} 0.0025 \mathrm{~mol} \mathrm{~L}^{-1}, \mathrm{MgSO}_{4} 0.002 \mathrm{~mol} \mathrm{~L}^{-1}$ e Ca $\left(\mathrm{H}_{2} \mathrm{PO}_{4}\right)_{2} 0.005 \mathrm{~mol} \mathrm{~L}^{-1}$. Ao final de cada extração, o excesso de solução no solo foi removido por sucção, a partir da instalação de bomba a vácuo que era conectada à parte inferior do tubo. Após isso, os tubos foram vedados com rolha de borracha para evitar a evaporação da água. Realizou-se uma extração inicial e outras após períodos sucessivos de 2, 2, 4, 4, 4, 4 e 4 semanas, totalizando 24 semanas de incubação. A determinação do $\mathrm{N}$ mineralizado foi realizada por meio de um espectrofotômetro UV-Vis, utilizando 4.0 e $3.5 \mathrm{~mL}$ da solução lixiviada para análise de amônio $\left(\mathrm{N}-\mathrm{NH}_{4}^{+}\right)$e nitrato $\left(\mathrm{N}-\mathrm{NO}_{3}{ }^{-}\right)$, respectivamente.

A mineralização de carbono da camada $0-10 \mathrm{~cm}$ do solo foi estimada pela quantidade de $\mathrm{CO}_{2}$ absorvida por uma solução de $\mathrm{NaOH}(10 \mathrm{~mL})$ contida em um recipiente de vidro que foi instalado na parte superior de cada coluna de percolação, conforme adotado por Araújo et al. (2001), Barreto et al. (2010) e Nunes et al. (2016). A solução de $\mathrm{NaOH}$ era trocada a cada período de incubação. A quantidade de $\mathrm{C}-\mathrm{CO}_{2}$ foi determinada por titulação da solução de $\mathrm{NaOH}$ com $\mathrm{HCl} 0.5 \mathrm{~mol} \mathrm{~L}^{-1}$.

A cinética de mineralização e os potenciais de mineralização de $N\left(N_{0}\right)$ e de carbono $\left(C_{0}\right)$ foram obtidos a partir dos valores acumulados de $\mathrm{N}$ mineralizado $\left(\mathrm{N}-\mathrm{NH}_{4}{ }^{+}\right.$e $\left.\mathrm{N}-\mathrm{NO}_{3}{ }^{-}\right)$e de $\mathrm{C}$ mineralizado $\left(\mathrm{C}^{-} \mathrm{CO}_{2}\right)$ durante as 24 semanas de incubação. Tais dados foram ajustados ao modelo sigmoidal (1), conforme adotado por Nunes et al. (2016), empregando-se o programa científico de ajuste de curvas SigmaPlot ${ }^{\circledR}$ v.12.0. 
Em que, $Y$ corresponde ao nitrogênio ou carbono mineralizado acumulado $\left(\mathrm{N}_{\mathrm{m}}\right.$ ou $\left.\mathrm{C}_{\mathrm{m}}\right)$ em $\mathrm{mg}$ $\mathrm{kg}^{-1}, \mathrm{X}_{\circ}$ o nitrogênio ou carbono potencialmente mineralizável $\left(\mathrm{N}_{\mathrm{o}}\right.$ ou $\mathrm{C}_{\mathrm{o}}$ ) em $\mathrm{mg} \mathrm{kg}^{-1}, k$ a constante de mineralização (semana $\left.{ }^{-1}\right), t_{0}$ o tempo inicial (semanas) e t o tempo de incubação (semanas).

A partir dos resultados de $\mathrm{N}_{0}, \mathrm{C}_{0}, \mathrm{~N}_{\text {total }}$ e COS, foram calculadas as relações $\mathrm{N}_{0}: \mathrm{N}_{\text {total, }} \mathrm{C}_{0}: \operatorname{COS}$ e $\mathrm{C}$ mineralizado/ $\mathrm{N}$ mineralizado $\left(\mathrm{C}: \mathrm{N}_{\min }\right)$.

\section{Suprimento de $\mathbf{N}$ pelas reservas orgânicas}

Os estoques de $\mathrm{N}$ do solo até a profundidade de $40 \mathrm{~cm}$ foram obtidos a partir da Expressão 2:

$E N=N * D s * E s p$

Em que: EN representa o estoque de $\mathrm{N}$ total do solo $\left(\mathrm{kg} \mathrm{ha}^{-1}\right), \mathrm{N}$ o teor de $\mathrm{N}$ total do solo em $\mathrm{g} \mathrm{kg}^{-1}$; Ds a densidade do solo em $\mathrm{g} \mathrm{cm}^{-3}$, e Esp a espessura da camada amostrada (cm).

Para estimar a participação das reservas orgânicas no suprimento de $\mathrm{N}$ para as plantações de eucalipto foram adotados dois modelos de predição, com base na metodologia proposta por Barreto et al. (2012) com adaptações, conforme descrito a seguir. Tais modelos foram aplicados para os valores médios das três rotações de cultivo, tendo em vista que não foram observadas variações significativas nos estoques de $\mathrm{N}$ do solo.

Modelo de predição I - Considerou o N potencialmente mineralizável do solo $\left(\mathrm{N}_{\mathrm{o}}\right)$ estimado para a profundidade 0-40 $\mathrm{cm}\left(\mathrm{kg} \mathrm{ha}^{-1} \mathrm{ano}^{-1}\right)$ e o estoque de $\mathrm{N}$ no sistema serapilheira-solo $\left(\mathrm{N}_{\mathrm{SES}}\right)$, que correspondeu ao somatório da quantidade média de $\mathrm{N}$ estocado na serapilheira acumulada $\left(\mathrm{N}_{\mathrm{SE}}\right)$ e no solo $\left(\mathrm{N}_{\mathrm{S}}\right)$ das plantações de eucalipto $\left(\mathrm{kg} \mathrm{ha}^{-1}\right)$. Para obter a quantidade de $\mathrm{N}$ da reserva orgânica do solo-serapilheira que é liberada com a mineralização a cada rotação $\left(\mathrm{N}_{\text {liberado }}\right)\left(\mathrm{kg} \mathrm{ha}{ }^{-1}\right.$ rotação $\left.{ }^{-1}\right)$, foi necessário estimar o fornecimento de $\mathrm{N}$ com a decomposição da serapilheira $\left(\mathrm{N}_{\mathrm{SE}}\right.$ fornecido $)\left(\mathrm{kg} \mathrm{ha}^{-1} \mathrm{ano}^{-1}\right)$, que foi somado ao $\mathrm{N}_{\mathrm{o}}$ e multiplicado pelo número de anos da rotação de cultivo $(r)$. O número de rotações supridas por estes compartimentos $(\mathrm{Nr})$ foi obtido com base no $\mathrm{N}_{\text {liberado }}$ e na exportação de $\mathrm{N}$ com a colheita $\left(\mathrm{N}_{\mathrm{ex}}\right)$, estimada de acordo com o $\mathrm{N}$ estocado no fuste com casca, considerando a manutenção dos demais componentes da biomassa arbórea na área ( $\mathrm{N}$ da biomassa vegetal, $\mathrm{N}_{\mathrm{BV}}=225.6 \mathrm{~kg}$ de $\mathrm{N}$ ha-1; $\mathrm{N}$ do lenho= 115.5 kg de $\mathrm{N} \mathrm{ha}^{-1}$ ). Assim, a predição foi obtida por meio das Equações 3 a 6.

$$
\begin{aligned}
& N_{S E S}=N_{S E}+N_{S} \\
& N_{\text {liberado }}=\left(N_{S E \text { fornecido }}+N_{o}\right) * r \\
& N_{S E \text { fornecido }}=N_{S E} * 0.4 \\
& N_{r}=N_{S E S} /\left(N_{\text {liberado }}+N_{e x}\right)
\end{aligned}
$$

A estimativa de $\mathrm{N}_{0}$ na profundidade $0-40 \mathrm{~cm}$ foi obtida a partir do $\mathrm{N}_{0}$ da camada $0-10 \mathrm{~cm}$, admitindo-se que a diminuição do $\mathrm{N}_{\mathrm{o}}$ nas camadas $10-20$ e 20-40 cm, em relação à camada 0-10 cm, corresponde a mesma porcentagem de redução verificada para o N total (7.6 e 18.4\%, respectivamente).

A taxa de decomposição de $40 \%$ considerada no cálculo do $\mathrm{N}_{\mathrm{SE}}$ fornecido (Equação 5), corresponde à média aritmética de diferentes taxas encontradas para eucalipto na literatura de referência: Costa et al. (2005) (30\%), Gama-Rodrigues \& Barros (2002) (40\%) e Pinto et al. (2016) (50\%), que estudaram, respectivamente, plantações de Eucalyptus grandis com 
5 e 8 anos de idade, de Eucalyptus grandis x Eucalyptus urophylla com 16 anos de idade e de Eucalyptus urophylla com cinco anos de idade.

Modelo de predição Il - Foi definido a partir da estimativa do balanço de $\mathrm{N}\left(\mathrm{N}_{\mathrm{B}}\right)$, Equaação 7, que correspondeu a diferença entre o estoque inicial de $N$ no sistema serapilheira-solo $(0-40 \mathrm{~cm})$ $\left(\mathrm{N}_{\mathrm{SES}}\right)$ e a exportação de $\mathrm{N}\left(\mathrm{N}_{\mathrm{ex}}\right)$. A partir deste resultado, a estimativa da duração do estoque do $N_{B}$ em número de rotações $\left(N r N_{B}\right)$ foi obtida através da relação entre $N_{B}$ e a demanda de $\mathrm{N}$ pelas árvores $\left(\mathrm{N}_{\mathrm{D}}\right)$, que corresponde a quantidade de $\mathrm{N}$ absorvida ( $\mathrm{N}$ acumulado na serapilheira a cada ano, considerando a primeira rotação $+\mathrm{N}$ acumulado na biomassa aérea das árvores, média das três rotações) (kg ha-1), conforme Fórmulas 7, 8 e 9.

$$
\begin{aligned}
& N_{B}=N_{S E S}-N_{e x} \\
& N r N_{B}=N_{B} / N_{D} \\
& N_{D}=N_{S E}+N_{B V}
\end{aligned}
$$

\section{Análises estatísticas}

Os dados obtidos foram analisados quanto à normalidade (Lilliefors) e homogeneidade (Cochran). Posteriormente, os dados foram submetidos à análise de variância segundo um delineamento inteiramente casualizado com quatro repetições $(\alpha=5 \%)$. Adotou-se o teste Tukey $(\alpha=5 \%)$ para comparação de médias. De forma complementar, foi realizada análise de correlação de Pearson entre o $\mathrm{N}$ mineralizado e o $\mathrm{N}$ estocado na biomassa total. As análises foram realizadas empregando-se o programa estatístico SAEG ${ }^{\circledR}$ v.9.1.

\section{RESULTADOS E DISCUSSÃO}

Ao final das 24 semanas de incubação, o valor acumulado de $\mathrm{N}$ mineralizado $\left(\mathrm{N}_{\mathrm{m}}\right)$ foi, em média, $45 \mathrm{mg}$ de $\mathrm{N} \mathrm{kg}^{-1}$ de solo, não havendo variação significativa entre as plantações de eucalipto e nem dessas em relação à floresta nativa (Tabela 2). Seguindo o mesmo padrão, as quantidades de $C$ mineralizado acumulado $\left(C_{m}\right)$ também não diferiram entre os tratamentos (Tabela 2), com média de $523 \mathrm{mg} \mathrm{kg}^{-1}$. Estes resultados sugerem que o tempo de cultivo de eucalipto, mesmo em uma condição de manejo incipiente de adubação, não afetou o potencial do solo de fornecer carbono e nitrogênio orgânico decomponível.

Tabela 2. Mineralização acumulada e potenciais de mineralização de $\mathrm{N}$ de solos cultivados com eucalipto

\begin{tabular}{|c|c|c|c|c|c|c|c|c|}
\hline \multirow{3}{*}{ Área } & \multirow{2}{*}{$\mathbf{N}_{\mathrm{m}}{ }^{\mathrm{ss}}$} & \multirow{2}{*}{$\mathbf{N}_{0}$} & \multirow{3}{*}{$\begin{array}{c}\mathbf{k}_{\mathrm{N}} \\
\text { semanas }^{-1}\end{array}$} & \multirow{3}{*}{$r^{2}$ No } & \multirow{3}{*}{$E P_{\text {No }}$} & \multicolumn{3}{|c|}{$N_{\text {total }}{ }^{\text {ns }}$} \\
\hline & & & & & & $0-10$ & $10-20$ & $20-40$ \\
\hline & \multicolumn{2}{|c|}{ - $\mathrm{mg} \mathrm{kg}^{-1}$ - } & & & & \multicolumn{3}{|c|}{ - - $\mathrm{kg} \mathrm{ha}^{-1}$ - } \\
\hline $\mathbf{R} 1$ & 46,17 & 49,90 & 4,84 & $0,99 *$ & 1,82 & 817,11 & 658,00 & 1049,60 \\
\hline $\mathbf{R 2}$ & 44,90 & 46,37 & 4,27 & $0,99 *$ & 1,95 & 1050,56 & 732,60 & 1378,00 \\
\hline R3 & 43,49 & 47,65 & 5,09 & $0,98 *$ & 1,97 & 933,83 & 691,60 & 962,00 \\
\hline FN & 45,31 & 46,37 & 4,11 & $0,99 *$ & 1,70 & 933,83 & 694,07 & 1129,87 \\
\hline Média & 44,97 & 47,57 & 4,58 & & & 116,73 & 37,36 & 219,31 \\
\hline $\pm S$ & 1,12 & 1,66 & 0,46 & & & 817,11 & 658,00 & 1049,60 \\
\hline
\end{tabular}
e sob floresta nativa, constante de mineralização $\left(k_{N}\right)$, estatísticas de ajuste ao modelo sigmoidal e estoque de nitrogênio total

R1: Eucalipto em primeira rotação de cultivo; R2: Eucalipto em segunda rotação, totalizando 9 anos de cultivo; R3: Eucalipto em terceira rotação, totalizando 14 anos de cultivo; $\mathrm{FN}$ : floresta nativa; $\mathrm{N}_{\mathrm{m}}$ : $\mathrm{N}$ mineralizado acumulado; ns: não significativo pelo teste Tukey a $5 \%$ de significância; $N_{0}$ : $N$ potencialmente mineralizável; $k_{N}$ : constante de mineralização de $\mathrm{N} ; \mathrm{r}^{2}{ }_{\mathrm{N}}$ : coeficiente de determinação do ajuste para a estimativa de $\mathrm{N}_{\circ} ; \mathrm{EP}_{\mathrm{No}}$ : erro-padrão da estimativa de $N_{0} ;{ }^{*} p<0,001 ; N_{\text {total: }}$ nitrogênio total estocado nas profundidades 0-10, 10-20 e 20-40 cm. 
A relação C:N mineralizado também não mostrou diferenças entre tratamentos, variando entre 11.3 e 13.2 (Tabela 3), o que denota que a capacidade de transformar C e N orgânicos em formas inorgânicas permaneceu a mesma, mesmo após três ciclos de cultivo de eucalipto. Estes valores são superiores aos encontrados por Nunes et al. (2016) (média de 4.9), próximos dos de Barreto et al. (2010) (média de 13.7) e inferiores aos de Araújo et al. (2001) (média de 24.4). Ou seja, o valor observado expressa uma eficiência intermediária do solo em converter formas orgânicas de N e C para formas inorgânicas.

Tabela 3. Relações N potencialmente mineralizável/ N total, C potencialmente mineralizável/ C orgânico do solo e $\mathrm{C} / \mathrm{N}$ mineralizado em áreas cultivadas com eucalipto a diferentes tempos e sob floresta nativa

\begin{tabular}{cccc}
\hline Áreas & $\mathbf{N}_{\mathbf{0}}: \mathbf{N t}$ & $\mathbf{C}_{\mathbf{o}}: \mathbf{C O S}$ & $\mathbf{C : N}_{\text {mineralizado }}{ }^{\text {ns }}$ \\
\hline R1 & 7,0 & 8,1 & 11,3 \\
R2 & 4,9 & 7,7 & 11,5 \\
R3 & 6,3 & 8,3 & 13,2 \\
FN & 3,6 & 4,3 & 11,6 \\
Média & 5,5 & 7,1 & 11,9 \\
$\mathbf{\text { S S }}$ & 1,5 & 1,9 & 0,9 \\
\hline
\end{tabular}

R1: Eucalipto em primeira rotação de cultivo; R2: Eucalipto em segunda rotação, totalizando 9 anos de cultivo;

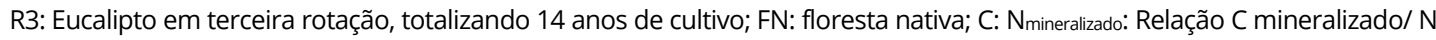
mineralizado; $\mathrm{N}_{0}$ : Nt: relação $\mathrm{N}$ potencialmente mineralizável/ $\mathrm{N}$ total; $\mathrm{C}_{0}$ : COS: relação C potencialmente mineralizável/ C orgânico do solo; ns: não significativo pelo teste Tukey a 5\% de significância.

O modelo sigmoidal (Figura 2) mostrou-se adequado para explicar a cinética de mineralização de $\mathrm{N}$ e de $\mathrm{C}$, gerando valores de regressão significativos e estimativas de $\mathrm{N}_{0}$ e $\mathrm{C}_{0}$ bem próximas dos valores de $\mathrm{N}$ e $\mathrm{C}$ mineralizados durante as 24 semanas de incubação (Tabela 2). Embora a maior parte das pesquisas sobre mineralização de $\mathrm{N}$ e $\mathrm{C}$ tenha constatado melhores ajustes para modelos exponenciais (Araújo et al., 2001; Barreto et al., 2010; Molina et al., 1980), Alves et al. (1999) e Nunes et al. (2016) observaram bom potencial preditivo do N e C mineralizado a partir do modelo sigmoidal. De acordo com Alves et al. (1999), o ajuste a este tipo de modelo resulta da ocorrência de desvios na cinética de primeira ordem, caracterizando padrão de mineralização que tende a uma curva sigmoidal. Essa variação na dinâmica de mineralização pode estar ligada a diversos fatores, como a atividade microbiana e a quantidade e qualidade da matéria orgânica do solo (Ameloot et al., 2015).
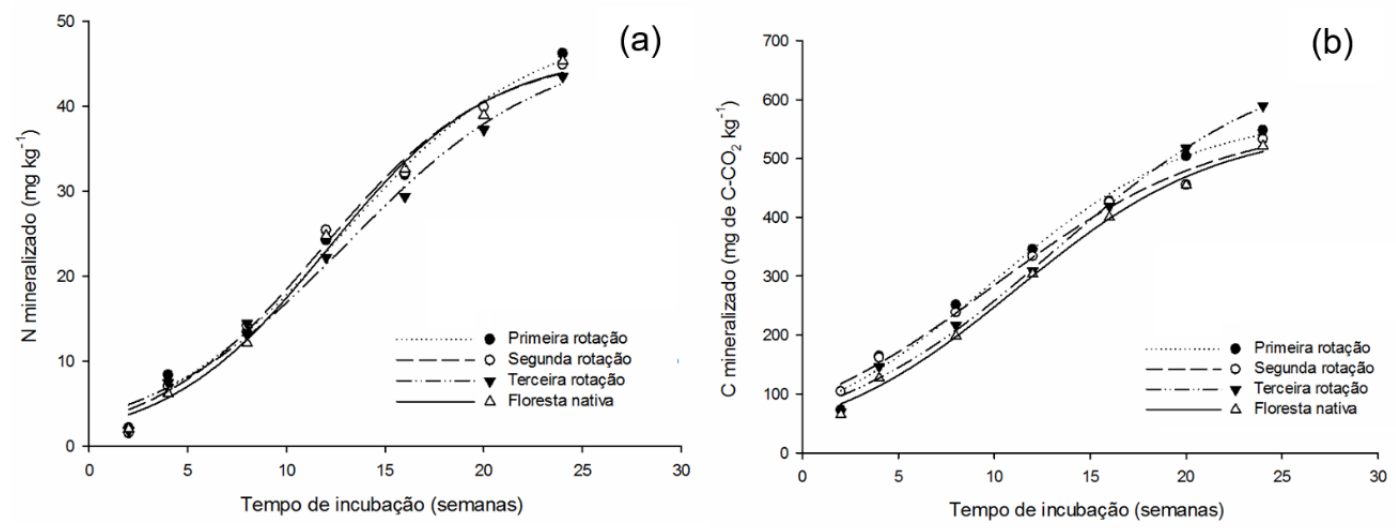

Figura 2. Curvas de mineralização acumulada de $\mathrm{N}(\mathrm{a})$ e de $\mathrm{C}$ (b) de solos cultivados com eucalipto e sob floresta nativa, ajustadas ao modelo sigmoidal para obtenção do potencial de mineralização.

As curvas de mineralização de $\mathrm{N}$ e de $\mathrm{C}$, obtidas a partir do ajuste do modelo sigmoidal (Figura 2) evidenciam uma liberação lenta de $\mathrm{N}$ mineral e de $\mathrm{CO}_{2}$ na fase inicial de incubação, seguida de aumentos progressivos, com padrão semelhante entre as áreas cultivadas com 
eucalipto e a floresta nativa. A partir da décima segunda semana, verificou-se queda da inclinação das curvas de todos os tratamentos, o que, segundo Pottker \& Tedesco (1979) e Camargo et al. (1997), indica que houve redução da quantidade de compostos orgânicos nitrogenados mais facilmente decomponíveis.

As quantidades de $\mathrm{N}_{\mathrm{o}}$ foram muito próximas entre as áreas de eucalipto e a floresta nativa, variando de 46.4 a $49.9 \mathrm{mg} \mathrm{kg}^{-1}$ (Tabela 2), o que significa que 3.6 a $7.0 \%$ do $\mathrm{N}$ total do solo é potencialmente mineralizável, considerando a relação $\mathrm{N}_{0}$ : Nt (Tabela 3). Resultados dentro desta mesma faixa de variação foram encontrados por Barreto et al. (2010) (entre 3.2 e 5.2\%) e Camargo et al. (2008) (5.0\%). A proximidade dos valores de $\mathrm{N}_{\mathrm{o}}$ entre as áreas estudadas indica que o cultivo de eucalipto por quase três rotações consecutivas (14 anos de cultivo) não alterou a reserva potencial de $\mathrm{N}$ orgânico decomponível do solo.

A constante de mineralização de $N\left(k_{N}\right)$ variou entre 4.1 semana $^{-1}$ (floresta nativa) e 5.1 semana-1 $^{-1}$ (terceira rotação) (Tabela 2). Isso demonstra que, embora não tenham se distinguido em relação a fração do $\mathrm{N}$ orgânico ativo, os solos apresentaram ligeira diferenciação em relação ao "turnover" microbiano. É provável que o destaque do valor de $K_{N}$ da terceira rotação esteja relacionado a maiores acúmulos de matéria orgânica, já que esta área apresentou serapilheira com estoques superiores de fitomassa $\left(26.0 \mathrm{Mg} \mathrm{ha}^{-1}\right.$ ) e de $\mathrm{N}$ (245.7 $\mathrm{Mg} \mathrm{ha}^{-1}$ ) em relação às demais áreas de eucalipto (fitomassa média $=14.6 \mathrm{Mg} \mathrm{ha}^{-1} \mathrm{e} \mathrm{N}$ estocado médio $=122.5 \mathrm{~kg}$ de $\mathrm{N} \mathrm{ha}^{-1}$ ), o que estaria favorecendo o estabelecimento de uma população microbiana mais eficiente no processo de mineralização.

Os resultados de carbono potencialmente mineralizável $\left(C_{0}\right)$ variaram entre 556.9 a $687.6 \mathrm{mg} \mathrm{kg}^{-1}$ (Tabela 4). Ao considerar a razão $\mathrm{C}_{0}$ : COS, observou-se valores de 4.3\%, para floresta nativa, e 8.0\%, para as rotações de cultivo (Tabela 3), o que evidencia que os solos sob eucalipto apresentam maior potencial relativo de mineralização de carbono. Nunes et al. (2016), estudando o efeito do cultivo de leguminosas florestais em solo com condição semelhante à do presente estudo, encontraram relação $C_{0}$ : COS média de $7.7 \%$.

Tabela 4. Mineralização acumulada e potenciais de mineralização de $C$ de solos cultivados com eucalipto e sob floresta nativa, constante de mineralização $\left(k_{c}\right)$, estatísticas de ajuste ao modelo sigmoidal e carbono orgânico do solo

\begin{tabular}{|c|c|c|c|c|c|}
\hline \multirow{2}{*}{ Área } & $C_{m}^{n s}$ & $\mathrm{C}_{\mathrm{o}}$ & \multirow{2}{*}{$\frac{k_{c}}{\text { semanas }^{-1}}$} & \multirow{2}{*}{$r^{2} c_{0}$} & \multirow{2}{*}{ EP co } \\
\hline & \multicolumn{2}{|c|}{$-\mathrm{mg} \mathrm{kg}^{-1}-$} & & & \\
\hline R1 & 509,58 & 578,10 & 5,26 & $0,99 *$ & 21,97 \\
\hline R2 & 518,63 & 566,97 & 5,91 & $0,99 *$ & 16,73 \\
\hline R3 & 559,97 & 687,63 & 6,15 & $0,99 *$ & 17,36 \\
\hline FN & 503,52 & 556,12 & 5,26 & $0,99 *$ & 14,31 \\
\hline Média & 522,92 & 597,21 & 5,65 & & \\
\hline $\pm S$ & 25,47 & 60,95 & 0,46 & & \\
\hline
\end{tabular}

R1: Eucalipto em primeira rotação de cultivo; R2: Eucalipto em segunda rotação, totalizando 9 anos de cultivo; R3: Eucalipto em terceira rotação, totalizando 14 anos de cultivo; FN: floresta nativa; $\mathrm{Cm}_{\mathrm{m}}$ : $\mathrm{C}$ mineralizado acumulado; ns: não significativo pelo teste Tukey a $5 \%$ de significância; $C_{0}$ : C potencialmente mineralizável; $k c$ - constante de mineralização de $C ; r^{2} C_{0}$ : coeficiente de determinação do ajuste para a estimativa de $C_{0}$; $E P_{c_{0}}$ : erro-padrão da estimativa de $C_{0} ;{ }^{*} p<0.001$.

Os valores de $C_{0}$ expressam que os solos apresentam grande potencial de mineralização de C. No entanto, o fato do potencial relativo de todas as áreas ter sido inferior a $10 \%$ (Tabela 3) é indicativo de que apenas uma pequena fração das reservas de $C$ orgânico do solo é decomponível. Isso sugere um favorecimento da acumulação de $C$ na camada superficial do solo, possivelmente associada à uma baixa decomposição da matéria orgânica. Dentre os fatores que corroboram esta explicação, estão a baixa qualidade orgânica e nutricional da serapilheira, constatada em vários estudos em plantações de eucalipto (Costa et al., 2005; Gama-Rodrigues \& Barros, 2002; Pinto et al., 2016), e os reduzidos índices pluviométricos observados na região de estudo. Baixas taxas de transformação dos resíduos orgânicos como 
consequência da redução da precipitação são comumente relatadas na literatura (Lima et al., 2015; Pinto et al., 2016).

A média da constante de mineralização do $C\left(k_{c}\right)$ foi de 5.6 semanas $^{-1}$ (Tabela 4), com ligeira diferenciação do solo sob terceira rotação em relação aos demais tratamentos. Estes resultados corroboram com os de $k_{N}$, denotando que as dinâmicas de mineralização de $\mathrm{C}$ e $\mathrm{N}$ estão intimamente relacionadas.

Seguindo o mesmo padrão das quantidades acumuladas de $\mathrm{N}$ mineralizado (Tabela 2), o nitrogênio total estocado até $40 \mathrm{~cm}$ de profundidade do solo não apresentou variação entre as áreas de estudo (Tabela 1), correspondendo, em média, a $2757.8 \mathrm{~kg} \mathrm{ha}^{-1}$. Apesar disso, verificou-se correlação positiva significativa entre o $\mathrm{N}$ mineralizado do solo e o conteúdo de $\mathrm{N}$ da biomassa arbórea nas diferentes rotações $(r=0.78 ; p<0.05)$, o que evidencia uma estreita relação entre produtividade e disponibilização de $\mathrm{N}$ por meio do processo de mineralização.

A quantidade de $\mathrm{N}$ potencialmente mineralizável dos solos sob eucalipto correspondeu a $87.8 \mathrm{~kg} \mathrm{ha}^{-1} \mathrm{ano}^{-1}\left(81.6 \mathrm{mg} \mathrm{kg}^{-1}\right)$ na camada $0-20 \mathrm{~cm}$, atingindo $153.7 \mathrm{~kg} \mathrm{ha}^{-1} \mathrm{ano}^{-1}\left(105.5 \mathrm{mg} \mathrm{kg}^{-1}\right)$ na profundidade $0-40 \mathrm{~cm}$. Estes valores se aproximam dos encontrados por Gonçalves et al. (2001) em solos de textura média (profundidade $0-15 \mathrm{~cm}$ ) sob cultivo de eucalipto com adoção de adubação nitrogenada (média de $83.0 \mathrm{mg} \mathrm{kg}^{-1}$ ).

Com base no modelo de predição $\mathrm{I}$, a quantidade de $\mathrm{N}_{0}$ da profundidade $0-40 \mathrm{~cm}$, juntamente com as demais reservas de $\mathrm{N}$, seriam suficientes para atender a demanda das plantas de eucalipto por até três rotações sucessivas de cultivo, sem considerar a complementação de $\mathrm{N}$ na forma de adubação nitrogenada. Por meio do modelo de predição II, que considera o balanço de $\mathrm{N}$ com a exportação apenas do fuste com casca (a média do $\mathrm{N}$ estocado neste componente para as três rotações estudadas correspondeu a $\left.115.5 \mathrm{~kg} \mathrm{de} \mathrm{N} \mathrm{ha}^{-1}\right)$, estima-se que o estoque médio de $\mathrm{N}$ presente no sistema serapilheira-solo (até $40 \mathrm{~cm}$ de profundidade) seria suficiente para suprir a demanda de $\mathrm{N}$ pelo eucalipto por cerca de oito rotações de 4 anos, não se verificando redução dessa previsão com o aumento do tempo de cultivo. Isso demonstra a possibilidade de um melhor planejamento das adubações nitrogenadas em plantações de eucalipto, de modo a evitar desperdícios, reduzindo os custos de produção e ainda a poluição do solo e cursos d'água.

Os resultados encontrados neste trabalho revelam que o nitrogênio mineralizado no solo seria a maior ou uma das maiores fontes deste nutriente para as plantas, uma vez que, mesmo não recebendo adubação nitrogenada, as plantações avaliadas, apresentaram produtividade superior a $83 \mathrm{~m}^{3} \mathrm{ha}^{-1}$. Tal produtividade supera a observada por Silva et al. (2013) para Eucalyptus urophylla sob Latossolo Vermelho-Amarelo e precipitação média anual de $700 \mathrm{~mm}\left(69.2 \mathrm{~m}^{3} \mathrm{ha}^{-1}\right)$ e está dentro da faixa de valores encontrada por Santos et al. (2017) para Eucalyptus sp. com quatro anos de idade sob Latossolo Amarelo e precipitação entre 700 e $1.400 \mathrm{~mm}$ (entre 25.2 e $193.1 \mathrm{~m}^{3} \mathrm{ha}^{-1}$ ). Isso também é uma comprovação de que as reservas orgânicas do solo exercem importante papel no suprimento da demanda de N, o que explica a baixa resposta do eucalipto à adubação nitrogenada, comumente relatada por pesquisadores e empresas florestais.

\section{CONCLUSÕES}

Nas condições estudadas, o potencial de mineralização de nitrogênio e de carbono do solo não é alterado pelo cultivo de eucalipto em até três rotações consecutivas. As reservas orgânicas de nitrogênio do solo são suficientes para atender a demanda de $\mathrm{N}$ pelo eucalipto por um período de três a oito rotações de cultivo.

\section{REFERÊCIAS BIBLIOGRÁFICAS}

Alves, G. D. (1989). Mineralização de carbono e nitrogênio em 20 solos do Estado de Pernambuco e absorção de nitrogênio pelo sorgo (Sorghum bicolor L. Menchú.) (Dissertação de mestrado). Universidade

Federal Rural de Pernambuco, Recife. 
Alves, G. D., Sampaio, E. V. S. B., Salcedo, I. H., \& Silva, V. M. (1999). Potencial de mineralização de N e C em vinte solos de Pernambuco. Revista Brasileira de Ciência do Solo, 23(2), 245-256. http://dx.doi.org/10.1590/S0100-06831999000200008.

Ameloot, N., Sleutel, S., Das, K. C., Kanagaratnam, J., \& Neve, S. (2015). Biochar amendment to soils with contrasting organic matter level: effects on $\mathrm{N}$ mineralization and biological soil properties. Global Change Biology Bioenergy, 7(1), 135-144. http://dx.doi.org/10.1111/gcbb.12119.

Araújo, A. M. S., Sampaio, E. V. S. B., \& Salcedo, I. H. (2001). Mineralização de carbono e nitrogênio em amostras armazenadas de solo cultivado com cana-de-açúcar, ao longo de dez anos, com e sem fertilização nitrogenada. Revista Brasileira de Ciência do Solo, 25(1), 43-53. http://dx.doi.org/10.1590/S0100-06832001000100005.

Azevedo, G. B., Sousa, G. T. O., Barreto, P. A. B., \& Conceição Júnior, V. (2011). Estimativas volumétricas em povoamentos de eucaliptos sob regime de alto fuste e talhadia no sudoeste da Bahia. Pesquisa Florestal Brasileira, 31(68), 309-318. http://dx.doi.org/10.4336/2011.pfb.31.68.309.

Barreto, P. A. B., Gama-Rodrigues, A. C., Gama-Rodrigues, E. F., \& Barros, N. F. (2012). Nitrogen balance in soil under eucalyptus plantations. Revista Brasileira de Ciência do Solo, 36(4), 1239-1248. http://dx.doi.org/10.1590/S0100-06832012000400018.

Barreto, P. A. B., Gama-Rodrigues, E. F., Gama-Rodrigues, A. C., Barros, N. F., Alves, B. J. R., \& Fonseca, S. (2010). Mineralização de nitrogênio e carbono em solos sob plantações de eucalipto, em uma sequência de idades. Revista Brasileira de Ciência do Solo, 34(3), 735-745. http://dx.doi.org/10.1590/S0100-06832010000300015.

Barros, N. F., Neves, J. C. L., \& Novais, R. F. (1990). Fertilização e correção do solo para o plantio de eucalipto. In N. F. Barros \& R. F. Novais (Eds.), Relação solo-eucalipto (pp. 127-186). Viçosa, Folha de Viçosa.

Bataglia, O. C., Furlani, A. M. C., Teixeira, J. P. F., Furlani, P. R., \& Gallo, J. R. (1983). Métodos de análise química de plantas (Boletim Técnico, No. 78). Campinas: Instituto Agronômico.

Brasil. (1994, 3 de fevereiro). Resolução CONAMA n 1, de 31 de Janeiro de 1994. Diário Oficial [da] República Federativa do Brasil (pp. 1684-1685), Brasília, seção 1.

Camargo, F. A. O., Gianello, C., \& Vidor, C. (1997). Potencial de mineralização do nitrogênio em solos do Rio Grande do Sul. Revista Brasileira de Ciência do Solo, 21(4), 575-579. http://dx.doi.org/10.1590/S0100-06831997000400007.

Camargo, F. A. O., Silva, L. S., Gianello, C., \& Tedesco, M. J. (2008). Nitrogênio orgânico do solo. In G. A. Santos, L. S. Silva, L. P. Canellas \& F. A. O. Camargo (Eds.), Fundamentos da matéria orgânica do solo: ecossistemas tropicais e subtropicais (2. ed., pp. 87-99). Porto Alegre: Metrópole.

Costa, G. S., Gama-Rodrigues, A. C., \& Cunha, G. M. (2005). Decomposição e liberação de nutrientes da serapilheira foliar em povoamentos de Eucalyptus grandis no Norte Fluminense. Revista Árvore, 29(4), 563-570. http://dx.doi.org/10.1590/S0100-67622005000400008.

Defelipo, B. V., \& Ribeiro, A. C. (1981). Análise química do solo: metodologia (Boletim de Extensão, No. 29). Viçosa: UFV.

Dijkstra, F. A., Jenkins, M., Courcelles, V. R., Keitel, C., Barbour, M. M., Kayler, Z. E., \& Adams, M. A. (2017). Enhanced decomposition and nitrogen mineralization sustain rapid growth of Eucalyptus regnans after wildfire. Journal of Ecology, 105(1), 229-236. http://dx.doi.org/10.1111/1365-2745.12663.

Empresa Brasileira de Pesquisa Agropecuária - EMBRAPA. Serviço Nacional de Levantamento e Classificação de Solos. (2017). Manual de métodos de análise de solo. Brasília: Embrapa.

Fernandes, J. S., Conceição Júnior, V., \& Barreto-Garcia, P. A. B. (2018). Field performance of Eucalyptus hybrids at Planalto da Conquista, Bahia, Brazil. Floresta e Ambiente, 25(2), 1-8. http://dx.doi.org/10.1590/2179-8087.059416.

Gama-Rodrigues, E. F., \& Barros, N. F. (2002). Ciclagem de nutrientes em floresta natural e em plantios de eucalipto e de dandá no Sudeste da Bahia. Revista Árvore, 26(2), 193-207.

Gama-Rodrigues, E. F., Barros, N. F., Gama-Rodrigues, A. C., \& Santos, G. A. (2005). Nitrogênio, carbono e atividade da biomassa microbiana do solo em plantações de eucalipto. Revista Brasileira de Ciência do Solo, 29(6), 893-901. http://dx.doi.org/10.1590/S0100-06832005000600007.

Gonçalves, J. L. M., Mendes, K. C. F. S., \& Sasaki, C. M. (2001). Mineralização de nitrogênio em ecossistemas florestais naturais e implantados do estado de São Paulo. Revista Brasileira de Ciência do Solo, 25(3), 601-616. http://dx.doi.org/10.1590/S0100-06832001000300009.

Indústria Brasileira de Árvores - IBÁ. (2017). Relatório IBÁ 2017. Brasília: IBÁ. 
Instituto Brasileiro de Geografia e Estatística - IBGE. (2012). Manual técnico da vegetação brasileira: sistema fitogeográfico, inventário das formações florestais e campestres, técnicas e manejo de coleções botânicas, procedimentos para mapeamentos (Manuais Técnicos de Geociências, No. 1). Rio de Janeiro: Diretoria de Geociências, IBGE.

Lima, R. P., Fernandes, M. M., Fernandes, M., \& Matricardi, E. A. T. (2015). Aporte e decomposição da serapilheira na Caatinga no Sul do Piauí. Floresta e Ambiente, 22(1), 42-49. http://dx.doi.org/10.1590/2179-8087.062013.

Molina, J. A. E., Clapp, C. E., \& Larson, W. E. (1980). Potentially mineralizable nitrogen in soil: the simple exponential model does not apply for the first 12 weeks of incubation. Soil Science Society of America Journal, 44(2), 442-443. http://dx.doi.org/10.2136/sssaj1980.03615995004400020054x.

Nunes, D. A. D., Gama-Rodrigues, E. F., Barreto, P. A. B., Gama-Rodrigues, A. C., \& Monroe, P. H. M. (2016). Carbon and nitrogen mineralization in soil of leguminous trees in a degraded pasture in northern Rio de Janeiro, Brazil. Journal of Forestry Research, 27(1), 91-99. http://dx.doi.org/10.1007/s11676-015-0164-3.

Pinto, H. C. A., Barreto, P. A. B., Rodrigues, E. F. G., Oliveira, F. G. R. B., De Paula, A., \& Amaral, A. R. (2016). Decomposição da serapilheira foliar de floresta nativa e plantios de Pterogyne nitens e Eucalyptus urophylla no Sudoeste da Bahia. Ciência Florestal, 26(4), 1141-1153. http://dx.doi.org/10.5902/1980509825105.

Pottker, D., \& Tedesco, M. J. (1979). Efeito do tipo e tempo de incubação sobre a mineralização da matéria orgânica e nitrogênio total em solos do Rio Grande do Sul. Revista Brasileira de Ciência do Solo, 3, 20-24.

Pulito, A., Gonçalves, J. L. M., Smethurst, P. J., Arthur Junior, J. C., Alvares, C. A., Rocha, J. H. T., Hubner, A., Moraes, L. F., Miranda, A. C., Kamogawa, M. Y., Gava, J. L., Chaves, R., \& Silva, C. R. (2015). Available nitrogen and responses to nitrogen fertilizer in brazilian eucalypt plantations on soils of contrasting texture. Forests, 6(12), 973-991. http://dx.doi.org/10.3390/f6040973.

Sanquetta, C. R., Corte, A. P. D., Balbinot, R., \& Zilliotto, M. A. B. (2004). Proposta metodológica para quantificação e monitoramento do carbono estocado em florestas plantadas. In C. R. Sanquetta \& M. A. B. Zilliotto (Eds.), Carbono: ciência e mercado global (pp. 120-150). Curitiba: UFPR/ECOPLAN.

Santos, A. C. A., Silva, S., Leite, H. G., \& Cruz, J. P. (2017). Influência da variabilidade edafoclimáticas no crescimento de clones de eucalipto no Nordeste baiano. Pesquisa Florestal Brasileira, 37(91), 259268. http://dx.doi.org/10.4336/2017.pfb.37.91.1207.

Silva, J. A. A., Rocha, K. D., Ferreira, R. L. C., \& Tavares, J. A. (2013). Produtividade volumétrica de clones de Eucalyptus spp. no polo gesseiro do Araripe, Pernambuco. Anais da Academia Pernambucana de Ciência Agronômica, 10, 240-260.

Stanford, G., \& Smith, S. J. (1972). Nitrogen mineralization potentials of soils. Soil Science Society of America Journal, 36(3), 465-472. http://dx.doi.org/10.2136/sssaj1972.03615995003600030029x.

Tchichelle, S. V., Epron, D., Mialoundama, F., Koutika, L. S., Harmand, J. M., Bouillet, J. P., \& Mareschal, L. (2017). Differences in nitrogen cycling and soil mineralisation between a eucalypt plantation and a mixed eucalypt and Acacia mangium plantation on a sandy tropical soil. Southern Forests, 79(1), 1-8. http://dx.doi.org/10.2989/20702620.2016.1221702.

Contribuição dos autores: AMO: conceituação, curadoria de dados, análise formal, investigação, escrita primeira redação; PABBG: conceituação, curadoria de dados, análise formal, metodologia, supervisão, administração do projeto, escrita - revisão e edição; BJRA: curadoria de dados, metodologia, recursos; VCJ: metodologia, recursos, escrita - revisão e edição; EFGR: conceituação, metodologia, recursos. 